\title{
Airfoil-Gust Interactions in Transonic Flow
}

\author{
James Gill, ${ }^{*}$ Xin Zhang, ${ }^{\dagger}$ Siyang Zhong, ${ }^{\ddagger}$ Ryu Fattah,,${ }^{\S}$ and David Angland ${ }^{\llbracket}$ \\ *, ${ }^{\top}$ Airbus Noise Technology Centre, \\ Faculty of Engineering and the Environment, \\ University of Southampton, Hampshire, SO16 7QF, UK. \\ $\dagger,,^{\ddagger} \S$ Department of Mechanical and Aerospace Engineering, \\ The Hong Kong University of Science and Technology, \\ Clear Water Bay, Kowloon, Hong Kong SAR, China.
}

\begin{abstract}
Leading edge noise is a significant broadband noise source in aircraft engines, and is the primary broadband noise mechanism in outlet guide vane noise in turbofans, and broadband rotor wake interaction noise in contra-rotating open rotor engines. Previous authors have studied the effects of various aspects relating to this noise source, including airfoil geometry effects, cascade effects, and Mach number effects. However, previous literature has not addressed the effects on the noise due to locally supersonic regions that might be present in the mean flow around the rotor blades. The current work uses computational aeroacoustic methods to investigate the effects of locally supersonic regions on the noise due to airfoilgust interactions. An established computational aeroacoustics code has been extended to give stable predictions in supersonic regions with a localized artificial diffusivity method. Initial results of a NACA 0012 airfoil in $M=0.8$ flow interacting with oncoming vortical waves are shown, alongside results for a NACA 0006 airfoil in $M=0.5$ flow at a $6^{\circ}$ angle of attack. The changes to the noise and the underlying mechanisms are discussed for both cases, including additional noise sources caused by the supersonic region.
\end{abstract}

Keywords: computational aeroacoustics, leading edge noise, acoustic shock interaction, shock capture

\section{Introduction}

$\mathrm{T}$ HE noise produced by interactions between an airfoil and oncoming vortical disturbances (usually called leading edge noise) is a significant broadband noise source in turbofan and contra-rotating open rotor engines (CRORs). In these engine designs, turbulence is generated upstream and then interacts with the leading edge of the outlet guide vane (OGV) in turbofans, or with the rear rotor in CRORs. The significance of leading edge noise has led to various previous works aiming to understand, measure, and predict this noise source. The long term goal is to provide methods that mitigate the aircraft noise and enable quieter flight.

In practice, leading edge noise from a realistic engine is a complicated and physically rich process that is affected by aspects such as the three-dimensional geometry of the blade, the statistics and inhomogeneity of the turbulence, rotational and forward flight speeds, and cascade effects. Therefore, studies to improve understanding of leading edge noise typically consider simplified situations, such as isolated blade interactions

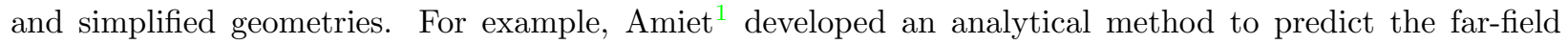

\footnotetext{
*Research Fellow, AIAA Member, J.Gill@soton.ac.uk.

† Swire Professor of Aerospace Engineering, Associate Fellow, AIAA, aexzhang@ust.hk.

${ }^{\ddagger} \mathrm{PhD}$ Student

$\S$ Research Fellow

ฯ Associate Professor, AIAA Member
} 
leading edge noise from a flat plate. Although the geometry of the airfoil was neglected, Amiet's theory forms the basis of current industrial prediction models of broadband leading edge noise. ${ }^{2}$ It is therefore desirable to understand the implications of any assumptions that are made in broadband leading edge noise studies so that the accuracy of industrial models can be determined.

Another simplification in previous studies of broadband leading edge noise is the consideration of lower freestream speeds, or lower angles of attack $(\mathrm{AoA})$, than those that appear on real configurations. For example, when investigating the effects of real CROR blade geometries on the noise, Gill ${ }^{3}$ chose an artificially reduced AoA in order to avoid effects caused by locally supersonic regions of flow. This paper describes a computational aeroacoustic (CAA) study to understand the effect of locally supersonic regions on the interactions between an airfoil and oncoming vortical gusts.

Studies have been performed on isolated airfoils at low speeds to investigate the effects on leading edge noise due to geometrical aspects such as airfoil thickness, leading edge radius, camber, and AoA. For example, Paterson and Amiet ${ }^{4}$ measured the noise from an isolated NACA 0012 airfoil at zero AoA, and showed that there is a reduction in high frequency leading edge noise due to the airfoil thickness when compared with analytical predictions of the noise from a flat plate. Similar conclusions were reached experimentally by Olsen and Wagner, ${ }^{5}$ Roger and Moreau, ${ }^{6}$ and Paruchuri et al.,$^{7}$ for example. The effects of airfoil thickness were also studied computationally by Atassi et al. ${ }^{8}$ Lockard and Morris, ${ }^{9}$ Gill et al. ${ }^{10,11}$ and Gea-Aguilera et $a l .{ }^{12}$ using a simplified turbulent input represented by a series of discrete frequency sinusoidal vortical waves. Similar behavior was found in the computational studies compared with the experimental measurements. The presence of thickness will reduce the noise at high frequency, and will alter the directivity pattern such that it is skewed in an upstream direction (i.e. sound reductions are greater in the downstream direction than in the upstream direction). Gill et al. ${ }^{10}$ suggested that this noise reduction is related to the distortion of vortices in the leading edge stagnation region.

Studies have also quantified the effects on the noise due to changes in the AoA. It has been generally concluded by authors that the effects of AoA are minimal (reported by Devenport as less than a $1 \mathrm{~dB}$ change in noise between $\alpha=0^{\circ}$ and $12^{\circ}$ for a NACA 0012 airfoil), see for example Paterson and Amiet, ${ }^{4}$ Moreau et al. ${ }^{13}$ Staubs, ${ }^{14}$ and Devenport et al. ${ }^{15}$ Similarly, Devenport et al. ${ }^{15}$ Moreau et al.,${ }^{13}$ and Gill et al. ${ }^{11}$ also showed that the noise is insensitive to changes in airfoil camber.

The variation of airfoil geometry effects on the noise, with changes in Mach number, was addressed by Paruchuri et al. ${ }^{7}$ In this study, the change in sound power level (PWL) in comparison to a flat plate was calculated for different airfoil geometries, and the study was repeated at various Mach numbers. It was shown that the effects of airfoil thickness appear to scale with Mach number, such that the PWL reduction for a given airfoil family will scale with $U / f$. This behavior was observed up to the highest tested freestream Mach number of $M=0.6$, and thus included compressibility effects. However, this relationship can be expected to break down at sufficiently high speeds, when additional aerodynamic physics in the flow, such as shocks, will begin to have an effect.

To the authors' knowledge, the study by Paruchuri et al. ${ }^{7}$ considered the highest Mach numbers to date for computational studies of leading edge noise of real airfoils. The computational tests were conducted at up to $M=0.6$ (experimental tests were conducted at up to $M=0.24$ ). The effects of higher velocities on leading edge noise have not been addressed. In real configurations where leading edge noise can occur, such as on the rear blades of an open rotor engine, regions of locally supersonic flow can occur. ${ }^{3,16,17}$ It is known that leading edge noise is highly sensitive to the aerodynamic meanflow surrounding the airfoil. Therefore, it is expected that high-speed flow features may have an effect on the noise.

This paper describes a computational aeroacoustic (CAA) study to understand the effect of locally supersonic regions on the interactions between an airfoil and oncoming vortical gusts. The presence of a locally supersonic region adds considerable difficulty to the aeroacoustic modeling process. For example, numerical schemes that are optimized for low-dispersion and low-dissipation must receive additional numerical treatment for stability in the presence of strong meanflow gradients. Entropy waves must also be considered in addition to the acoustic and vortical waves that are usually present in leading edge noise problems. Interactions that are usually assumed to be linear can now be non-linear. Finally, as is shown in this paper, the presence of a locally supersonic region can cause noise sources in addition to the traditional leading edge source. This can be due to deformation of vortical waves by the strong meanflow gradients, for example. Therefore, the effects of the locally supersonic region on leading edge noise must be investigated, and the additional noise sources that are caused by the locally supersonic region must also be considered. 


\section{Problem Description}

The interaction between single frequency vortical gusts and airfoils will be considered here. Two isolated airfoil configurations are chosen. The first configuration consists of a NACA 0012 airfoil placed at zero angle of attack (AoA) in a $M=0.8$ flow. The second is a NACA 0006 airfoil at $\mathrm{AoA}=6^{\circ}$ in a $M=0.5$ flow. The first configuration is intended to provide a simple configuration for initial analysis. The second is more representative of the conditions on a rotor blade. A schematic of the configuration is shown in Figure 1. It can be difficult to isolate the effects on the noise due to the supersonic region, since it is difficult to remove this region without significantly altering the case. The NACA 0012 case is compared to predictions from a NACA 0002 airfoil at $M=0.8$, and to predictions from a NACA 0012 airfoil at $M=0.7$ in order to identify the effects of the supersonic region. Similarly, the NACA 0006 case is compared to predictions from a NACA 0018 airfoil at $M=0.5$, which has sufficiently smooth curvature to generate a subsonic meanflow.

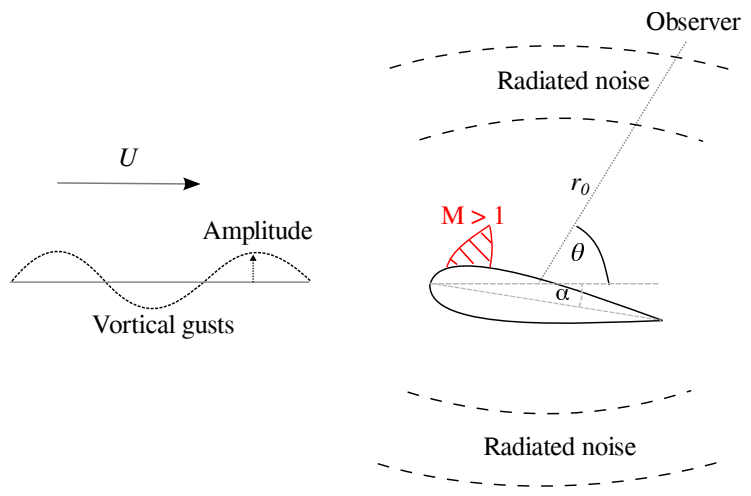

Figure 1: Schematic of the airfoil-gust interaction configuration, where $U$ is the freestream speed, $r_{0}$ is the observer radius, $\theta$ is observer angle, and $\alpha$ is the airfoil AoA.

Single frequency vortical gusts have been chosen to allow for indentifying and highlighting major physics in the study. The general simulation approach consists of two parts. A steady state simulation is performed to establish the steady mean flow surrounding the airfoil. Then, vortical gusts are introduced and propagate towards, and interact with, the isolated airfoil.

\section{Numerical Methods}

An established computational aeroacoustics (CAA) code is chosen to perform the simulations. This code has been previously used to study duct mode radiation, ${ }^{18}$ high lift device noise ${ }^{19}$ landing gear noise,${ }^{20}$ and the leading edge noise of isolated airfoils. ${ }^{10,11,12}$ It is able to solve the linearized Euler equations (LEEs), the full Euler equations, or the Navier-Stokes equations. In this study the full Euler equations are solved. In contrast to several previous works on leading edge noise, the linearized Euler equations cannot be used here since the presence of shock waves can introduce non-linear behavior. The use of inviscid governing equations is a simplification. However, previous leading edge noise simulations have shown that the effects of viscosity are small. ${ }^{9,10,21}$ Viscous flow effects may be larger when considering cases with locally supersonic regions, as the position of shocks may be affected, for example. However, in this preliminary study viscosity is neglected in order to reduce the complexity and computational cost.

The CAA code solves the Euler equations using high-order schemes originally designed for low-dispersion and low-dissipation characteristics. A fourth-order implicit spatial scheme ${ }^{22}$ is combined with a compact filter with variable cut-off wavenumber ${ }^{23}$ to provide high spatial resolution. Temporal resolution is achieved with a third-order Runge-Kutta scheme. ${ }^{24}$ The primitive variables are non-dimensionalized by $\rho=\rho^{*} / \rho_{0}$, $u=u^{*} / c_{0}, v=v^{*} / c_{0}$, and $p=p^{*} /\left(\rho_{0} c_{0}^{2}\right)$, where $\rho, u, v$, and $p$ represent non-dimensional variables, the subscript 0 represents a freestream quantity, the superscript ${ }^{*}$ represents a dimensional quantity, and where $c_{0}$ is the freestream speed of sound.

In this study, the interaction between an isolated airfoil and single frequency transverse vortical gusts is 
considered. The vortical waves are described by

$$
v(\boldsymbol{x}, t)=v_{\text {gust }} \cos \left(k_{x}(\boldsymbol{x}-U t)\right)
$$

where $U$ is the freestream flow speed, $v_{\text {gust }}$ is the gust amplitude (set to $0.01 U$ ), $k_{x}$ is the transverse vortical gust wavenumber, and $t$ is time.

To permit the study of supersonic flow regions, a localized artificial diffusivity (LAD) scheme ${ }^{25}$ has been implemented to provide numerical stability around flow discontinuities such as shocks. This is an adaptation of the method proposed by Cook, ${ }^{26}$ which adds artificial fluid transport coefficients to the physical transport coefficients (which are zero for Euler simulations) in the regions surrounding flow discontinuities. The shock is identified by using a combination of the negative dilatation and a Ducros-type sensor. This shock sensor enables the addition of artificial viscosity in regions of strong flow compression near shock waves, but disables it in expansion regions and weakly compressible regions. Some dimensionless user-specified constants are required for this type of shock wave treatment. The values of these constants were chosen to be the same as used by Kawai et al..$^{25}$ The implementation of the LAD method is validated in Section IV.

It is common in leading edge noise studies to predict the far-field noise. For example, Gill et al. ${ }^{11}$ used data from the airfoil surface in a Ffowcs-Williams and Hawkings (FW-H) solver to predict far-field noise. However, this method assumes radiation of the sound through a uniform flow, which is likely to be inaccurate when the potential refraction effects of the shock are considered. Therefore, off-surface data collection would be more appropriate for far-field noise predictions here. However, off-surface collection also experiences issues in the current simulations, as the forced vortical wave can be misinterpreted as sound and will contaminate the predictions. To avoid this issue, the current work will investigate only the local sound field at an observer radius of up to $r_{0}=4 \mathrm{~m}$ from the airfoil mid-chord.

Structured grids are used in this study. The domain extends to 8 chord lengths from the airfoil in all directions, similarly to Kim et al. ${ }^{27}$ The vortical gusts are over-resolved with at least 15 points per wavelength upstream of the airfoil to minimize any grid-induced errors. An additional requirement is that any regions involving shockwaves are also well resolved. At least 7 points have been used across the shock waves in the current work.

The computational grids used in this study utilize a sharp trailing edge geometry. Additional numerical treatment is required at the trailing edge due to the use of slip-wall boundary conditions on the airfoil surface. Along the majority of the airfoil surface, the velocity is forced to be tangential to the wall. However, at the trailing edge point the velocity is forced to be tangential to the mean chord-line of the airfoil. This method was found to significantly reduce numerical discontinuities seen at the trailing edge. However, some small errors remain in this region, caused by the abrupt change in velocity direction and by the abrupt change from wall condition to free-field condition just downstream of the trailing edge. It is likely that a viscous simulation would remove these errors, since the flow would be zero at the wall.

\section{Validations of Local Artificial Dissipation Method}

\section{IV.A. Stationary Normal Shock}

To provide initial validation of the LAD method implementation, a stationary normal shock case was computed as previously given by Kawai et al. ${ }^{28}$ This case considers a $M=2$ stationary normal shock wave that corresponds to a pressure jump across the shock of $p_{d} / p_{u}=4.5$, where $p_{u}$ and $p_{d}$ represent the pressure upstream and downstream of the shock, respectively. A uniform grid spacing of $\Delta x=\Delta y=0.005 \mathrm{~m}$ was used in a two-dimensional computational domain that extended from $-0.5<x<0.5 \mathrm{~m}$ and $0<y<0.5 \mathrm{~m}$. The normal shock was placed at $x=0.01 \mathrm{~m}$. In this case, the shock was resolved across 7 grid points.

Figure 2 shows the pressure jump in the domain due to the shock, as computed with and without the LAD treatment. It can be seen that the LAD treatment significantly smooths the pressure response across the shock wave, and appears to almost completely remove any oscillations in the shock region. Additionally, the LAD treatment does not affect the size of the pressure jump. This result is in close agreement with results presented by Kawai et al.,${ }^{25}$ and therefore serves as an initial validation of the implementation in the current work. 


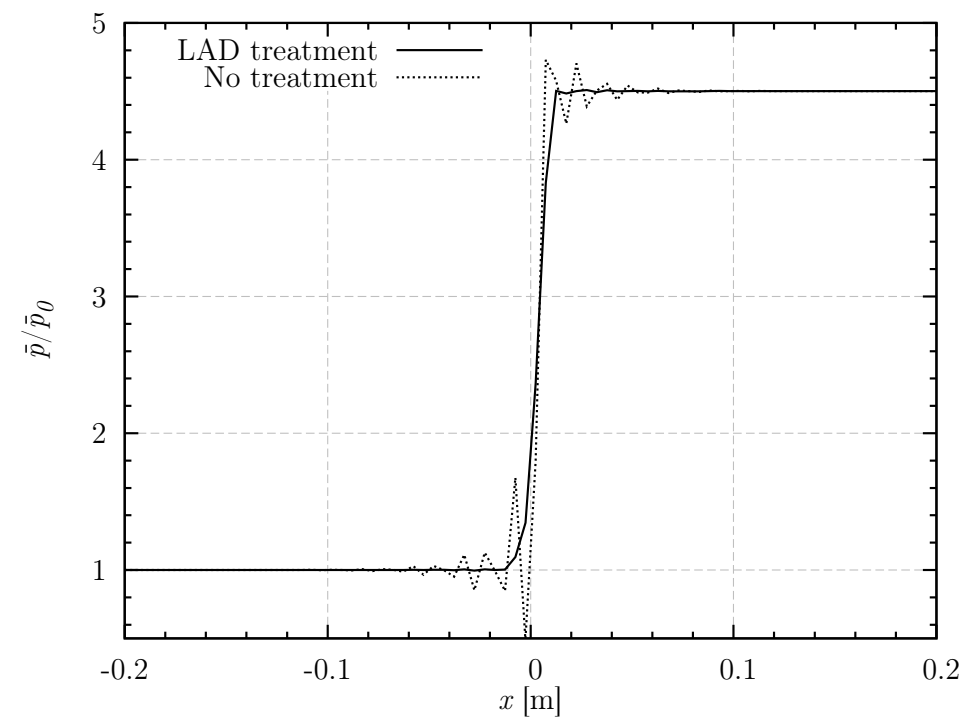

Figure 2: Steady non-dimensional pressure response along the streamwise centre-line due to a stationary normal shock at $M=2$.

\section{IV.B. Two-Dimensional Transonic Nozzle}

To provide further validation, a two-dimensional transonic nozzle was used as a more complex validation case. This case is Problem 2 in Category 1 in the "Third CAA Workshop on Benchmark Problems" ${ }^{29}$ and was previously considered by Kim and Lee. ${ }^{30}$ The chosen numerical method provided a good match with the steady and unsteady benchmark results, and with those from Kim and Lee. However, for brevity, the results of this case are omitted here. It was concluded that the chosen numerical method is capable of accurately modelling the interactions between an acoustic wave and a shock.

\section{Leading Edge Noise Predictions in Transonic Flow}

The described numerical method is applied to two transonic airfoil configurations in this section. Additional cases are also used to simulate leading edge noise in similar configurations, but without locally supersonic flow, in order to demonstrate the effects of the locally supersonic regions.

\section{V.A. NACA 0012 Airfoil}

The NACA 0012 airfoil was simulated at $M=0.7$ and $M=0.8$. The $M=0.8$ meanflow speed is sufficient to generate a localized region of supersonic flow that terminates in a normal shock wave approximately halfway along the airfoil chord. The mean pressure and velocity magnitude around the airfoil are shown in Figure 3 for the $M=0.8$ case. The amplitude of the vortical gust is $v_{\text {gust }}=0.01 U$, and the wavelength of the vortical gust is $\lambda=0.5 \mathrm{~m}$ (giving a reduced frequency $K=c / \lambda$ of 2 , where $c=1 \mathrm{~m}$ is the airfoil chord). Two additional gust wavelengths of $\lambda=1.0 \mathrm{~m}$ and $\lambda=1.5 \mathrm{~m}$ were also tested for the NACA 0012 airfoil case. The first stage of the computation produced a steady mean flow, and the second stage then generated a single frequency vortical plane wave gust upstream of the airfoil, which propagated towards and then interacted with the airfoil leading edge. 

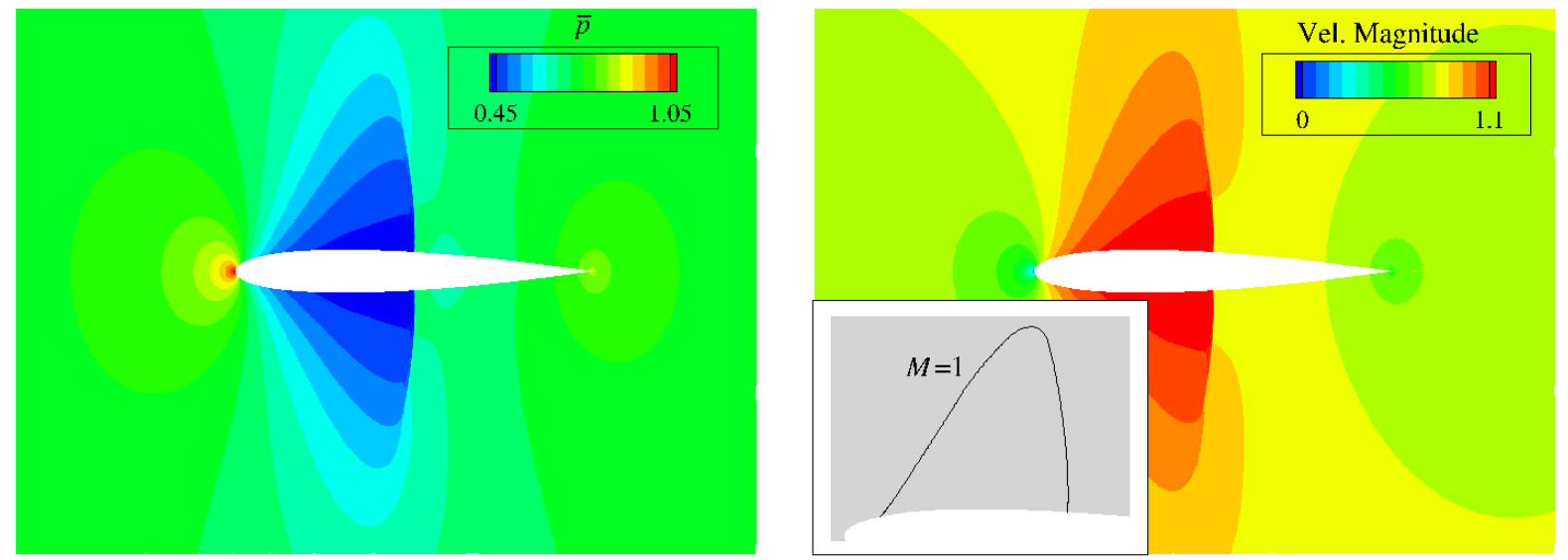

Figure 3: Steady pressure and velocity magnitude flow fields around a NACA 0012 airfoil at $M=0.8$. Magnified area shows the contour of $M=1$.

\section{V.A.1. Surface Pressure}

The steady pressure response on the NACA 0012 airfoil surface is shown in Figure 4. The shock is well captured, with only small oscillations in the pressure response immediately upstream and downstream of the shock. The mean surface pressure from the current study is compared with a solution from the QZSFLOW3D code $^{31}$ in Figure 4, which shows a good agreement. The pressure fluctuations on the airfoil surface are also shown in Figure 4. Strong pressure fluctuations are seen at the leading edge, similarly to the results of previous studies. ${ }^{4}$ However, the strongest $p^{\prime}$ response is seen at the chordwise location of the shock. This may represent an additional noise source that is not seen in subsonic cases. Alternatively, Kim and Lee ${ }^{30}$ found a similar spike in acoustic pressure in their simulation of an unsteady nozzle flow, and attributed this to shock oscillation. A small peak in $p^{\prime}$ can also be seen at the trailing edge. The reasons for this peak were discussed earlier, and it is not thought to have a significant effect on the noise predictions.

\section{V.A.2. Acoustic Field and Directivity}

The overall simulation is illustrated in Figure 5, which shows instantaneous contours of the acoustic pressure $p^{\prime}$, and sound pressure level (SPL), overlaid onto contours of velocity magnitude when $M>1$. This is done for both the NACA 0012 airfoil and for a NACA 0002 airfoil. The NACA 0002 airfoil has been included in order to provide comparison against a flow-field that does not include a shock wave. In general, the situation looks similar to previous studies that have been made at lower Mach numbers. The noise radiates from the airfoil as a non-compact dipole, with clearly visible acoustic radiation peaks, and with the strongest radiation being in the downstream direction. The noise radiated in the downstream direction is similar between the NACA 0002 and the NACA 0012 airfoils. However, in the upstream direction, the NACA 0012 airfoil radiates a larger number of well defined acoustic radiation peaks than are seen in the NACA 0002 case. These acoustic peaks appear to radiate from the edge of the supersonic region at approximately halfway along the airfoil chord. This effect has not been reported in previous works at lower Mach numbers, and is attributed to the supersonic flow region. Almost zero upstream acoustic radiation emanates from within the supersonic region because the acoustic waves cannot propagate upstream against a supersonic flow. The mechanism that causes the strong upstream radiation peaks is currently unknown. However, it may be partly due to a disruption in the back-scattering process from the trailing edge, or may simply be due to acoustic waves radiating from the leading edge and reflecting against the shock. Alternatively, this may be an additional noise source caused by interactions between vortical gusts and the supersonic region. Further investigation is needed.

Figure 6 shows Sound Pressure Level (SPL) directivities from a NACA 0002 and NACA 0012 airfoil interacting with vortical gust wavelengths of $\lambda=0.5,1.0$, and 1.5, and for flow speeds of $M=0.7$ and 0.8 . As an initial prediction, directivities have been recorded within the domain at an observer radius of $4 \mathrm{~m}$ from the airfoil mid-chord. The acoustic pressure was recorded for a total of 5 complete periods. Examination 

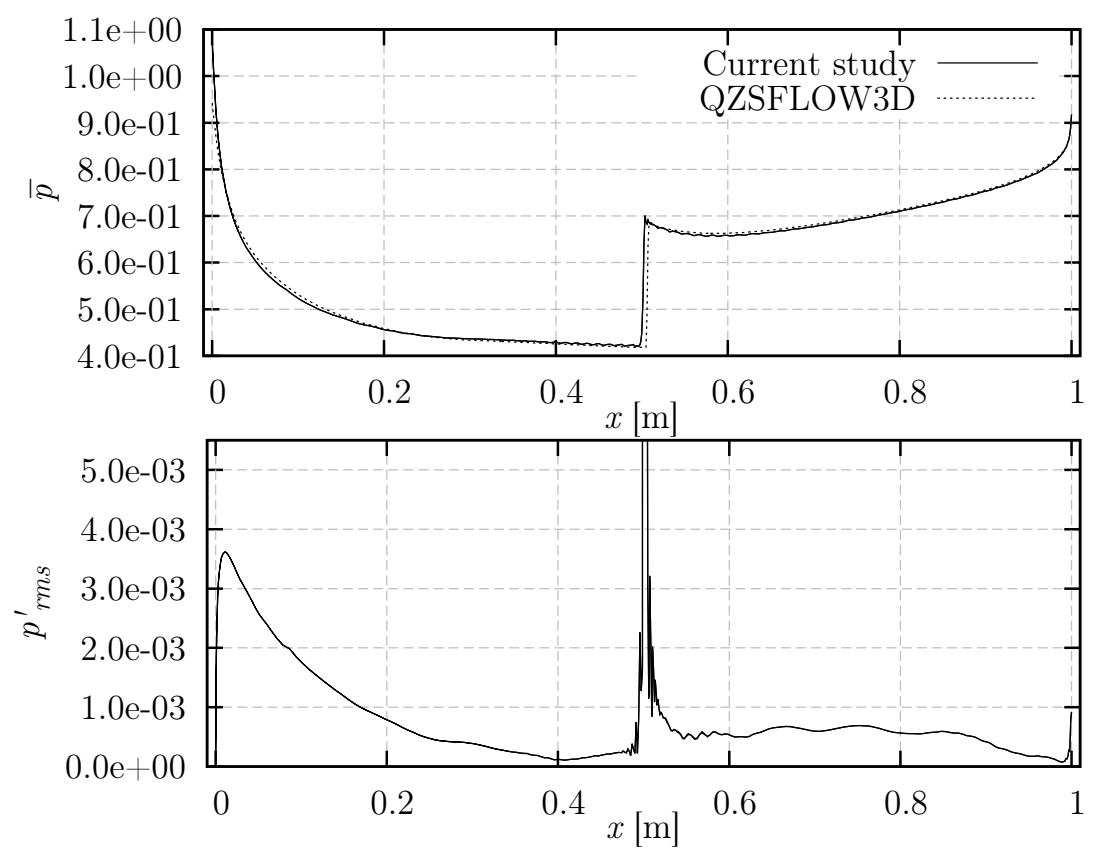

Figure 4: Variation of non-dimensional mean pressure $\bar{p}$ (top) and pressure fluctuations $p_{r m s}^{\prime}$ (bottom) along the NACA 0012 airfoil chord at $M=0.8$ with $\lambda=0.5 \mathrm{~m}$.

of the $M=0.8, \lambda=0.5 \mathrm{~m}$ case shows the behavior that was already identified in Figure 5 . The NACA 0012 case displays a much greater number of upstream acoustic radiation peaks than the NACA 0002 case. However, the overall amplitude levels are similar between the two airfoils, particularly in the downstream direction. This is because the chosen gust frequency is not sufficiently high to cause the geometry effects that have been identified in previous works, ${ }^{11}$ such as the reduction of noise in the downstream direction. It is expected that a higher frequency might demonstrate this behavior. However, a higher frequency was not tested here due to limitations in computational cost. This test therefore demonstrates that the effects of the supersonic region on the noise can occur at lower reduced frequencies than the effects of airfoil geometry.

The $M=0.7, \lambda=0.5 \mathrm{~m}$ case was chosen to provide comparison with a thick airfoil case that does not contain a supersonic region. Figure 6 shows that this case does not contain the strong additional upstream radiation peaks that are seen for $M=0.8$, which suggests again that these peaks are caused by the presence of the supersonic region. It should be noted that the same vortical wavelengths were used for the $M=0.7$ case. Therefore, the acoustic frequency of the noise is altered in comparison to the $M=0.8$ case, which will affect the directivity pattern. This explains the smaller number of radiation peaks that are seen in the $M=0.7$ case compared with results at $M=0.8$.

The $\lambda=1.0$ and $1.5 \mathrm{~m}$ simulations show similar behavior to the $\lambda=0.5 \mathrm{~m}$ case. At $M=0.8$, the NACA 0012 airfoil shows a greater number of strong acoustic radiation peaks than the NACA 0002, but does not display this behavior at $M=0.7$. Therefore, it appears that the effects of the supersonic region occur at multiple gust frequencies. However a study of frequency spectra is recommended to explore the frequency dependence in more detail. 

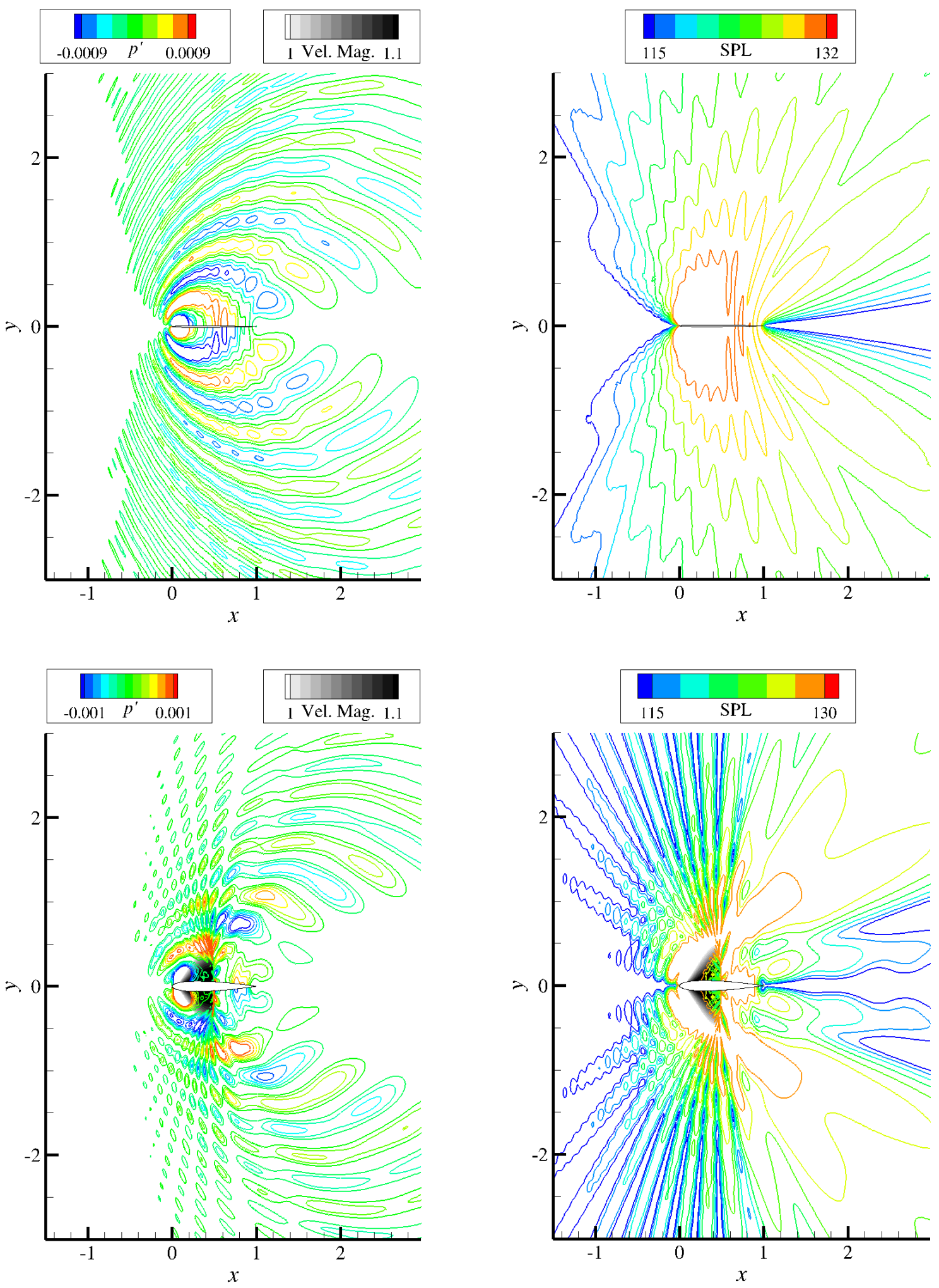

Figure 5: Instantaneous contours of acoustic pressure $p^{\prime}$ and SPL, overlaid onto contours of velocity magnitude when $M>1$, for a NACA 0002 (upper) and a NACA 0012 (lower) airfoil at $M=0.8$, with $\lambda=0.5$ m. 

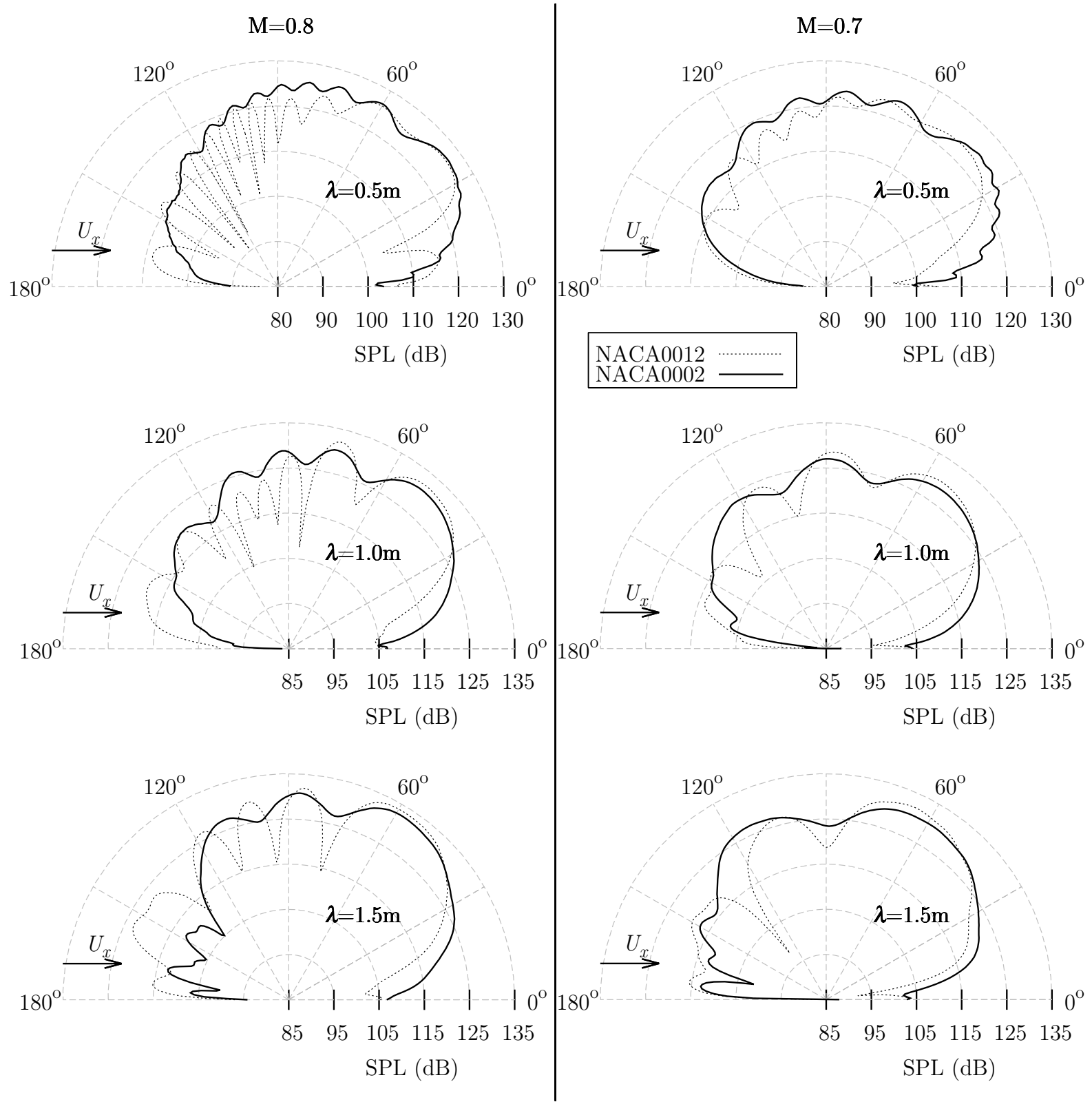

Figure 6: Acoustic directivity at $r_{0}=4 \mathrm{~m}$ for NACA 0012 and NACA 0002 airfoils in $M=0.7$ and $M=0.8$ flow, interacting with vortical gusts with wavelengths of $\lambda=0.5,1.0$, and $1.5 \mathrm{~m}$. 


\section{V.B. NACA 0006 Airfoil}

This section examines the noise and the flow-field surrounding a NACA 0006 airfoil placed at $\alpha=6^{\circ}$ in $M=0.5$ flow. Figure 7 shows contours of the steady pressure and velocity magnitude around the NACA 0006 airfoil. While the majority of the flow-field is subsonic, a small region of supersonic flow can be seen near the leading edge on the suction-side. In comparison to the NACA 0012 case, the supersonic region is considerably smaller here. However, the maximum local Mach number (of about $M=1.4$ ) is higher.
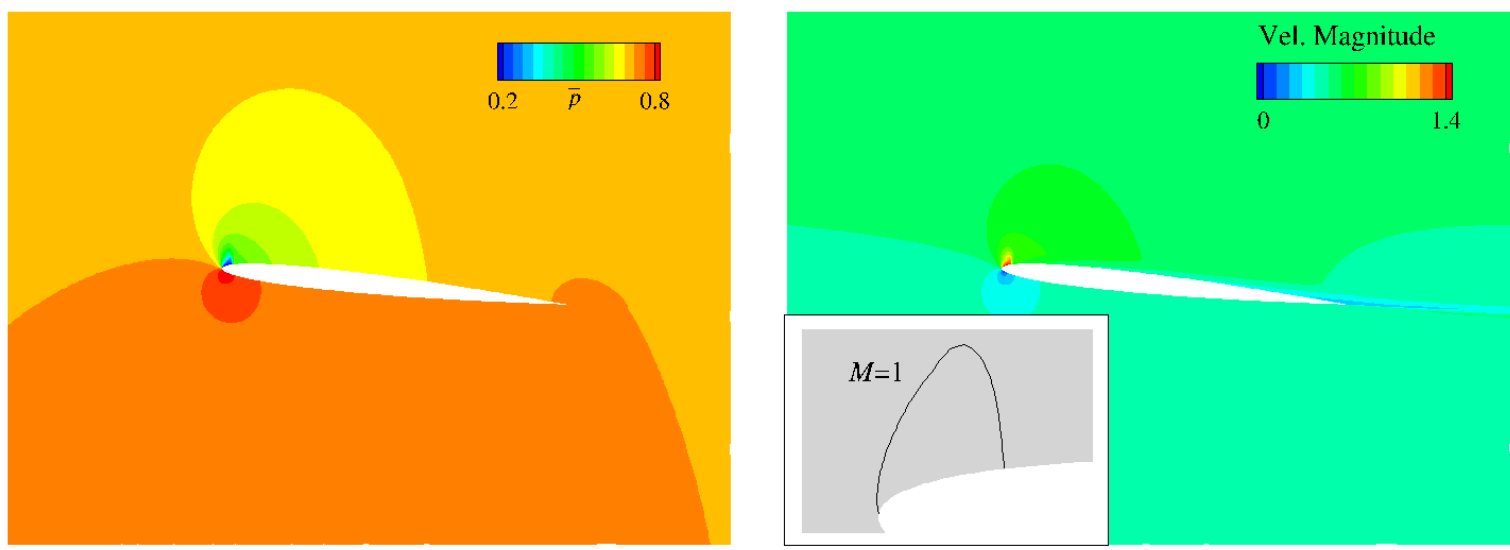

Figure 7: Steady pressure and velocity magnitude flow fields around a NACA 0006 airfoil at $M=0.5$ and $\alpha=6^{\circ}$. Magnified area shows the contour of $M=1$.

It is more difficult to determine the effects of the supersonic region in this case than for the NACA 0012 airfoil. Comparison of the NACA 0006 predictions with a flat plate (or very thin airfoil) prediction is not practical since the very sharp leading edge curvature would also lead to a locally supersonic flow region. Therefore, a NACA 0018 airfoil is also simulated at $M=0.5$ with $\lambda=0.5 \mathrm{~m}$. The NACA 0018 simulation provides a case that is just subsonic, thus removing the supersonic region. The significant difference in geometry between the $6 \%$ and $18 \%$ thick airfoils may have an effect on the noise. However, previous studies have found that effects on the noise due to changes in airfoil thickness are small for gusts with low reduced frequency, ${ }^{10}$ as is the case here $(K=c / \lambda=2)$.

\section{V.B.1. Interference from the Airfoil Wake}

The NACA 0006 case contains some additional modeling difficulties in comparison to the NACA 0012 case. The meanflow solution contains a region of decelerated flow near the trailing edge on the suction-side of the airfoil, which is shown in Figure 7. This was found to cause additional distortions to the vortical gusts as they pass over the trailing edge, generating spurious noise when the suction and pressure-side gusts interact in the wake region. This effect is shown by Figure 8, which shows contours of transverse gust velocity in the vicinity of the trailing edge. The meanflow solution was verified in several fluid solvers and is believed to be correct for the inviscid flow case considered here.

In order to minimize the impact of any spurious wake pressure on the leading edge noise predictions, additional numerical treatment was used where a sixth-order explicit filter was applied in the wake region. This filter is sufficiently strong to suppress any resulting noise from the wake, and to eventually damp the vortical waves far from the trailing edge. The effect of the additional filtering is shown in Figure 8. This noise source is likely to be a result of the inviscid flow assumption that is used here. In a viscous simulation it might not be present, or might behave differently. While it is a "physical" noise source for the Euler equations, in the sense that it is not the result of numerical error, it is undesirable here 

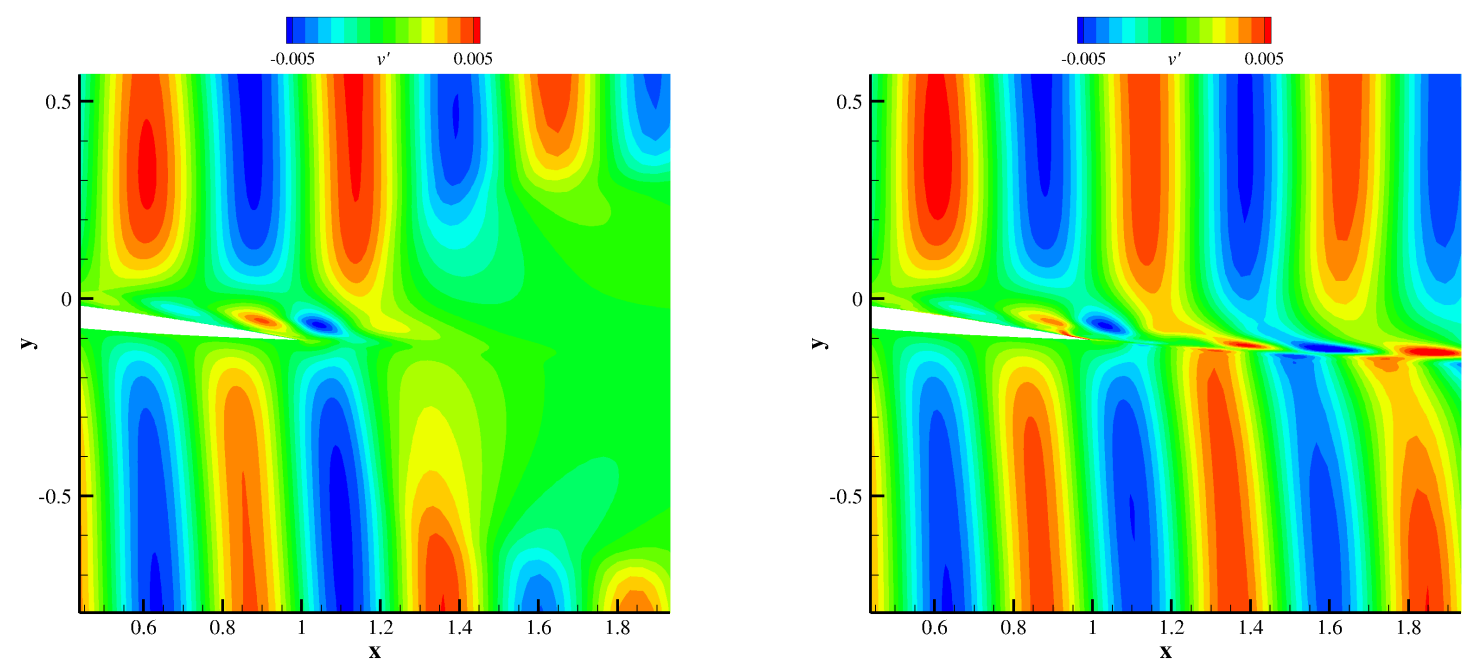

Figure 8: Contours of transverse velocity around the trailing edge of a NACA 0006 airfoil at $M=0.5$ and $\alpha=6^{\circ}$, simulated with (left), and without (right), additional filtering in the wake region.

\section{V.B.2. Surface Pressure}

Figure 9 shows the steady mean pressure and the $p_{\text {rms }}^{\prime}$ response to a $\lambda=0.5 \mathrm{~m}$ gust along the surface of the airfoil. As in the NACA 0012 case, the prediction has been compared with results from the QZSFLOW3D code, ${ }^{31}$ and a close agreement is seen. The presence of the supersonic region near the leading edge can be seen in the jump in steady pressure on the suction-side of the airfoil at about $x=0.04 \mathrm{~m}$. As was seen in the NACA 0012 case, this causes a corresponding spike in $p_{r m s}^{\prime}$ on the suction-side. The spike is located at the region of greatest $p_{r m s}^{\prime}$, and thus the location of the supersonic region appears to coincide with the location of strongest leading edge noise radiation. It is again unclear whether this spike represents a new noise source, or shows changes in the acoustic wave amplitude due to shock oscillation.
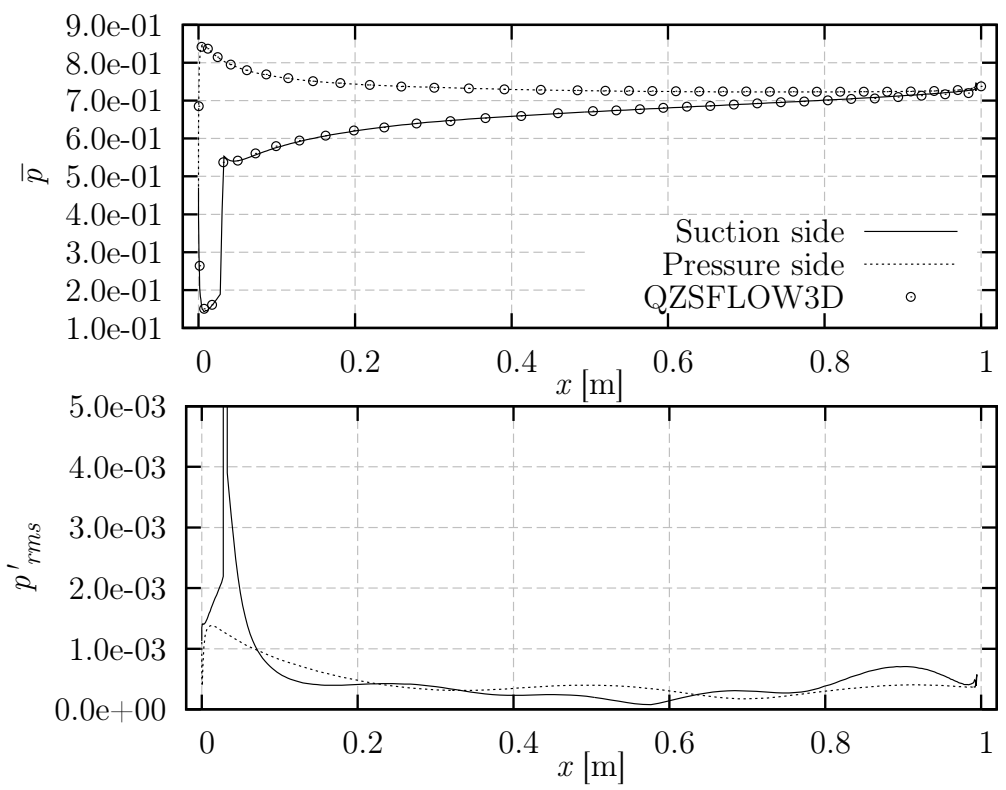

Figure 9: Variation of non-dimensional mean pressure $\bar{p}$ (top) and pressure fluctuations $p_{\text {rms }}^{\prime}$ (bottom) along the NACA 0006 airfoil chord at $M=0.5$. 


\section{V.B.3. Acoustic Field and Directivity}

Contours of instantaneous acoustic pressure $p^{\prime}$ and SPL are shown in Figure 10 for the NACA 0006 and NACA 0018 airfoils. Figure 10 shows that the non-zero AoA has affected the noise directivity such that it is no longer symmetrical about the streamwise axis. The strongest acoustic radiation peaks are in the upstream direction on the pressure-side of the airfoil, and in the downstream direction on the suction-side. The directivity around the NACA 0018 airfoil contains fewer peaks than the NACA 0006 airfoil. Strong upstream pressure-side, and downstream suction-side, acoustic peaks are still seen. However, the weaker peaks seen for the NACA 0006 case are not present for the NACA 0018 airfoil. It can therefore be concluded that changing the airfoil geometry has significantly altered the directivity pattern in this case. However, it is not clear whether this change is caused by the presence of the supersonic region or by the more common mechanisms that alter leading edge noise as the geometry of an airfoil is changed. These are discussed in the next section.

Figure 11 compares the SPL directivity for the NACA 0006 and NACA 0018 airfoil cases. The NACA 0006 airfoil is generally louder than the NACA 0018, which is likely caused by the difference in airfoil thickness. The most significant difference is seen at $\theta=120^{\circ}$, where an additional directivity lobe is present for the NACA 0006. Previous studies into the effects of airfoil thickness have reported that the directivity pattern is generally unchanged by a change in airfoil thickness. ${ }^{10,32}$ Therefore, this difference is thought to be caused by the supersonic region.

It should be noted that Gill et al. ${ }^{11}$ and Devenport et al. ${ }^{15}$ have shown that using single frequency harmonic gusts with streamwise wavenumber $k_{y}=0$, such as those used here, may not be sufficient to predict leading edge noise for airfoils at non-zero angle of attack. The current tests are useful to understand the mechanisms involved, but may not provide an accurate prediction of the full effects on leading edge noise. To obtain more accurate solutions a turbulence synthesis method should be used that contains vortical gust variations in both $k_{x}$ and $k_{y}$.

\section{V.B.4. Effects on the Vortical Gusts}

Previous authors, such as Gill et al. ${ }^{10}$ have found the mechanism that changes leading edge noise with airfoil geometry is linked to distortions in vortical gusts around the leading edge. Figure 12 compares contours of instantaneous gust velocity magnitude around the NACA 0006 and NACA 0018 airfoils. The NACA 0018 airfoil shows a similar behavior to previous studies on both the suction and pressure-sides of the airfoil. As gusts approach the leading edge, the wavefront is curved due to the passage of the gust through the leading edge stagnation region. In the NACA 0006 case, the presence of the suction-side supersonic region distorts the gust near to the airfoil surface and results in a more complex vortical wave pattern. The strongest levels of velocity magnitude are at the shock, and along the airfoil surface on the suction-side. It is not known why the perturbations are strongest near the wall. This behavior requires additional study, though it may be related to the strongest velocity gradients in the supersonic region, which will be closest to the wall. However, this behavior might not occur in a viscous simulation due to the presence of a boundary layer. Therefore, a fully viscous simulation may be necessary for improved noise predictions.

The strong values of velocity magnitude near the wall may affect the far-field directivity by altering the vortical gust interactions with the airfoil surface, and might also contribute to the wake noise that was previously discussed. Therefore, it seems likely that the supersonic region is affecting the noise of this case by altering the vortical wave as it interacts with the airfoil surface. However, this may not be the only effect of the supersonic region. For example, the supersonic region may also refract the emitted noise and thus alter the noise directivity. It should also be noted that the deformation of the vortical gusts by the shock wave may instead be the direct cause of the additional upstream directivity lobe. This deformation will likely violate the divergence-free condition of the gust, and may be an additional noise source. Further study of isolated interactions between vortical gusts and shocks is recommended to investigate this.

Figure 13 shows contours of mean velocity magnitude above $M=1$, overlaid with instantaneous gust velocity vectors (colored by gust velocity magnitude) for the NACA 0006 airfoil. This is done to display the effect of the supersonic region on the vortical gusts as they pass around the NACA 0006 airfoil. The vortical disturbances appear to be amplified in the streamwise direction as they pass through the shock at the rear of the supersonic region. This appears to be the cause of the gust deformation seen along the airfoil surface in Figure 12. 

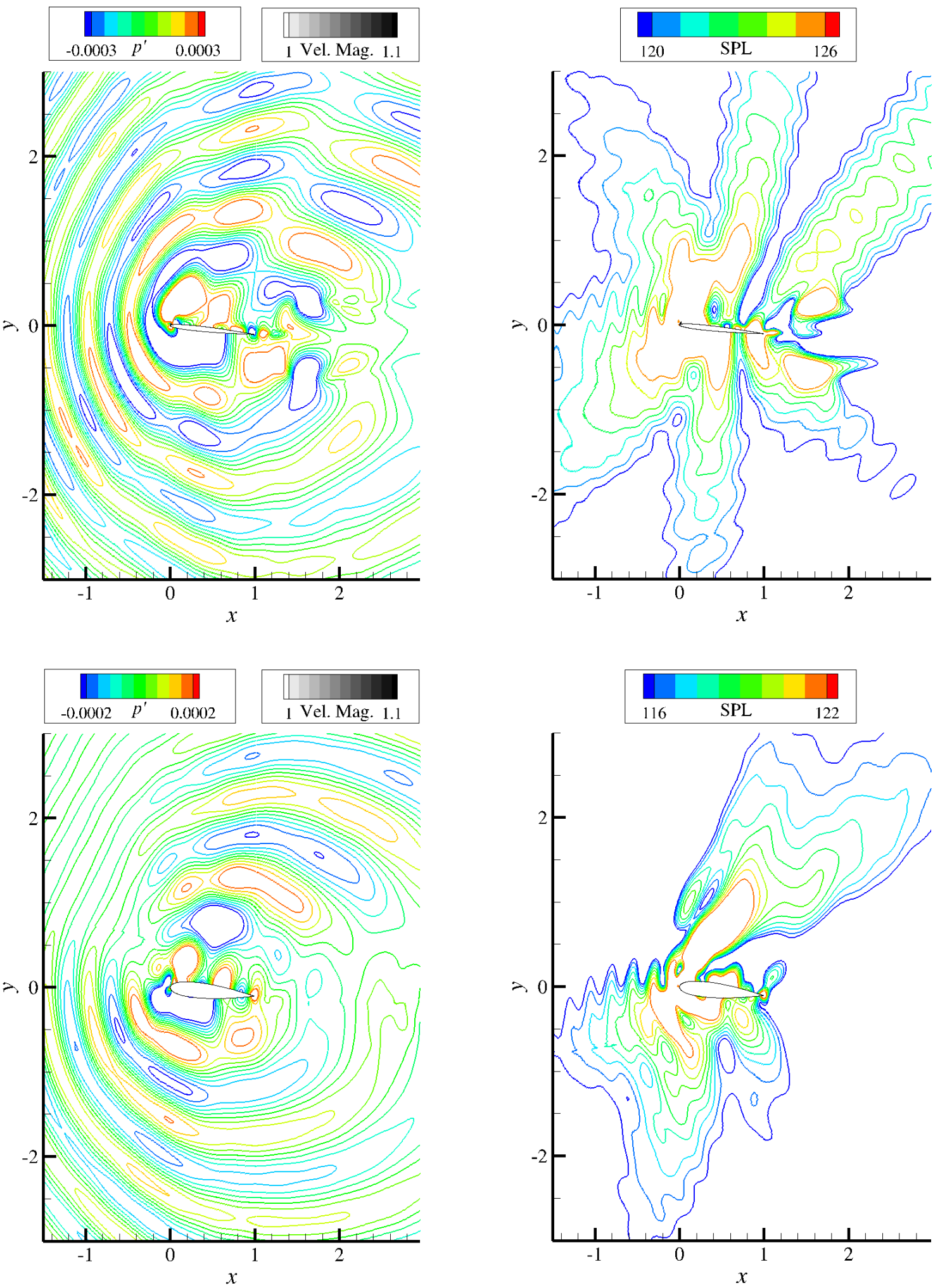

Figure 10: Contours of instantaneous acoustic pressure $p^{\prime}$ and SPL overlaid onto contours of velocity magnitude when $M>1$, for a NACA 0006 airfoil and a NACA 0018 airfoil at $M=0.5$. 
NACA 0006, $\mathrm{M}=0.5, \lambda=0.5 \mathrm{~m}$

NACA $0018, \mathrm{M}=0.5, \lambda=0.5 \mathrm{~m}$

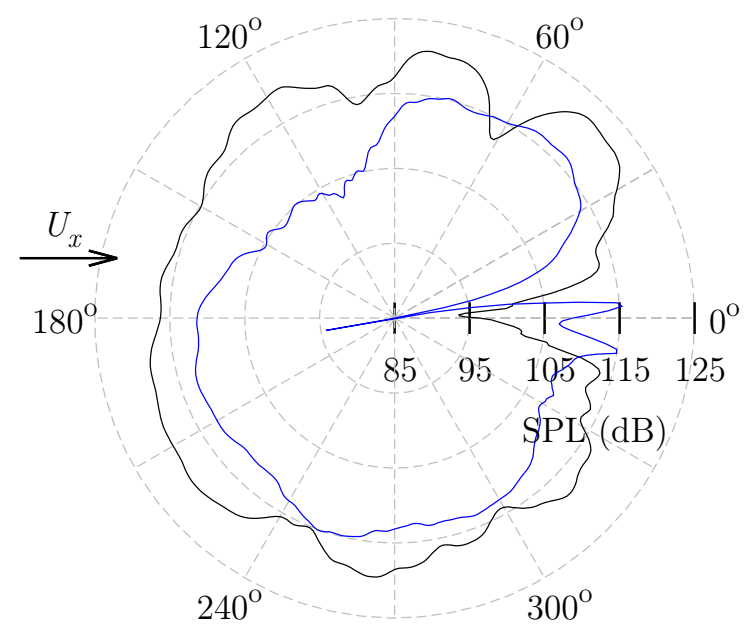

Figure 11: Variation of non-dimensional mean pressure $\bar{p}$ (top) and acoustic pressure $p^{\prime}$ (bottom) along the NACA 0006 airfoil chord at $M=0.5$ with $\lambda=0.5 \mathrm{~m}$.
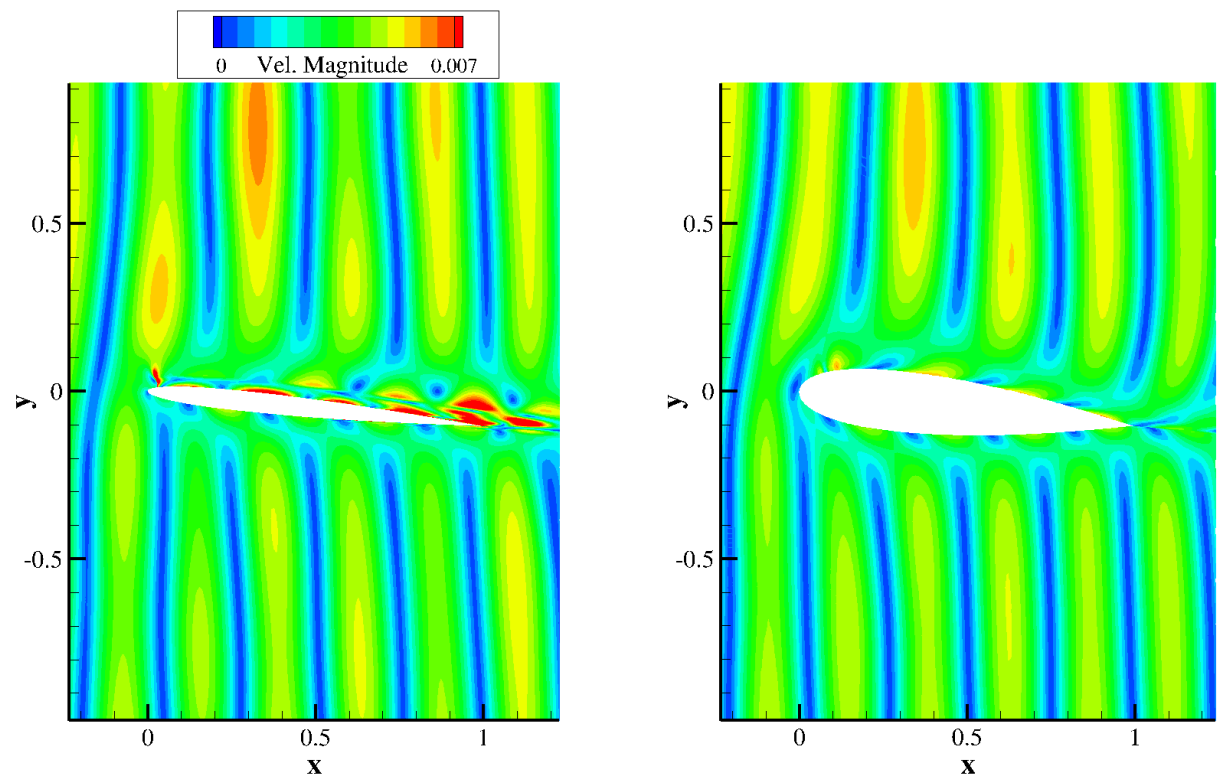

Figure 12: Contours of instantaneous non-dimensional gust velocity magnitude around a NACA 0006 airfoil (left) and a NACA 0018 airfoil (right) at $\alpha=6^{\circ}$ in $M=0.5$ flow.

\section{Summary}

Leading edge noise is a significant broadband noise source in turbofan and CROR engine designs. This noise source has been extensively studied by previous authors to investigate the effects of various aspects on the noise, such as airfoil geometry, cascade effects, Mach number, and the properties of the oncoming turbulence. In some situations, blade sections can experience locally supersonic regions of flow and the 


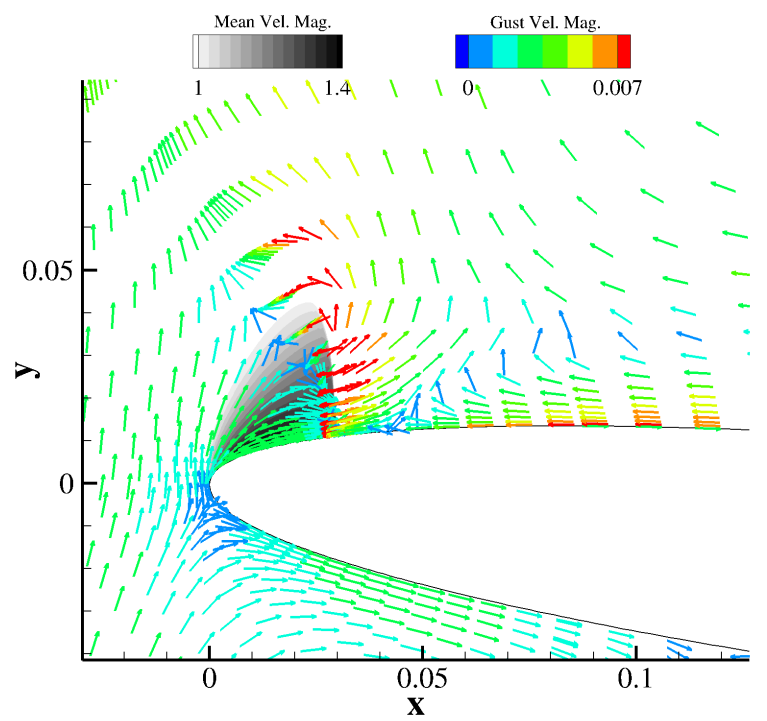

Figure 13: Contours of mean velocity magnitude (grayscale), overlaid with instantaneous gust velocity vectors colored by velocity magnitude, for the NACA 0006 airfoil. Quantities are non-dimensional.

effects of these regions on the noise have not been previously addressed. This paper has described a method to computationally investigate the effects of locally supersonic regions, and shock waves, on airfoil-gust interaction noise. A locally artificial dissipation scheme has been implemented and validated in an existing computational aeroacoustics solver to allow stable computations. Simulation results of a NACA 0012 airfoil at $M=0.8$, and a NACA 0006 airfoil at $M=0.5$ and $\alpha=6^{\circ}$, have been shown. The main conclusions are:

- The locally supersonic region alters the upstream directivity of the NACA 0012 airfoil when encountering vortical gusts. A larger number of well-defined acoustic radiation peaks are seen when the supersonic region is present.

- The effect of the supersonic region on the noise from the NACA 0012 airfoil occurs at multiple gust frequencies.

- The effects of the supersonic region can occur at reduced frequencies that are lower than the reduced frequency at which airfoil geometry effects on the noise become significant.

- The noise from the NACA 0006 airfoil exhibits an additional upstream directivity lobe in comparison to the NACA 0018 airfoil case. This may be caused by the presence of the supersonic flow region. However, it is difficult to isolate the effects of the supersonic region from those due to changes in the airfoil geometry.

- The exact cause of the changes in upstream acoustic radiation is unknown for both the NACA 0012 and NACA 0006 cases. Further investigation is needed to determine if the leading edge noise source is being modified by the presence of a locally supersonic flow region, or if the supersonic region is introducing additional noise sources.

- The amplitude of vortical gusts appears to be increased in the streamwise direction as they pass through the shock around the NACA 0006 airfoil. This effect distorts the vortical gust and may change the behavior of interactions with the airfoil surface.

- There are some additional modeling difficulties associated with the NACA 0006 airfoil case. The distortion of gusts by the shock, and the presence of mean velocity gradients near the trailing edge, cause an additional noise source in the wake region as the suction- and pressure-side gusts interact behind the airfoil. This source was suppressed in the current study.

The current study is an initial investigation into the leading edge noise of airfoils with locally supersonic regions. To simplify the analysis, single frequency harmonic gusts have been used, and directivities have 
been explored in the near-field using an analysis that solves the Euler equations. Future work to investigate airfoil noise behavior in the far-field, and with varying frequency, would be beneficial. It is also recommended to determine whether the modeling of viscosity is necessary for the NACA 0006 case.

\section{Acknowledgement}

The authors would like to thank Zhansen Qian for kindly providing the QZSFLOW3D code that is compared with in this work.

\section{References}

${ }^{1}$ Amiet, R. K., "Acoustic Radiation from an Airfoil in a Turbulent Stream," Journal of Sound and Vibration, Vol. 41, No. 4, 1975, pp. 407-420.

${ }^{2}$ Node-Langlois, T., Wlassow, F., Languille, V., Colin, Y., Caruelle, B., Gill, J., Chen, X., Zhang, X., and Parry, A. B., "Prediction of Contra-Rotating Open Rotor Broadband Noise in Isolated and Installed Configurations," 20th AIAA/CEAS Aeroacoustics Conference, Atlanta Georgia, No. 2014-2610, 2014 2015.

${ }^{3}$ Gill, J., Broadband Noise Generation of a Contra-Rotating Open Rotor Blade, Ph.D. thesis, University of Southampton,

${ }^{4}$ Paterson, R. W. and Amiet, R. K., "Noise and Surface Pressure Response of an Airfoil to Incident Turbulence," Journal of Aircraft, Vol. 14, No. 8, 1977, pp. 729-736.

${ }^{5}$ Olsen, W. and Wagner, J., "Effect of Thickness on Airfoil Surface Noise," AIAA Journal, Vol. 20, No. 3, 1981, pp. 437439.

${ }^{6}$ Roger, M. and Moreau, S., "Extensions and Limitations of Analytical Airfoil Broadband Noise Models," International Journal of Aeroacoustics, Vol. 9, 2010, pp. 273-305.

${ }^{7}$ Paruchuri, C., Gill, J., Narayanan, S., Joseph, P., Vanderwel, C., Zhang, X., and B. Ganapathisubramani, "Aerofoil geometry effects on turbulence interaction noise," 21st AIAA/CEAS Aeroacoustics Conference, Dallas TX, No. 2015-2830, 2015, pp. 1-20.

${ }^{8}$ Atassi, H. M., Dusey, M., and Davis, C. M., "Acoustic Radiation from a Thin Airfoil in Nonuniform Subsonic Flows," AIAA Journal, Vol. 31, 1993, pp. 1993.

${ }^{9}$ Lockard, D. and Morris, P., "Radiated Noise from Airfoils in Realistic Mean Flows," AIAA Journal, Vol. 36, No. 6, 1998, pp. 907-914.

${ }^{10}$ Gill, J., Zhang, X., and Joseph, P. F., "Symmetric airfoil geometry effects on leading edge noise." The Journal of the Acoustical Society of America, Vol. 134, No. 4, oct 2013, pp. 2669-80.

${ }^{11}$ Gill, J., Zhang, X., and Joseph, P. F., "Single Velocity-Component Modeling of Leading Edge Turbulence Interaction Noise," Journal of the Acoustical Society of America, Vol. 137, No. 6, 2015, pp. 3209-3220.

${ }^{12}$ Gea-Aguilera, F., Zhang, X., Chen, X., and Gill, J., "Synthetic Turbulence Methods for Leading Edge Noise Predictions," 21st AIAA/CEAS Aeroacoustics Conference, Dallas, No. 2015-2670, 2015.

${ }^{13}$ Moreau, S., Roger, M., and Jurdic, V., "Effect of Angle of Attack and Airfoil Shape on Turbulence Interaction Noise," 11th AIAA/CEAS Aeroacoustics Conference, Monterey, California, No. 2005-2973, 2005.

${ }^{14}$ Staubs, J. K., Real Airfoil Effects on Leading Edge Noise, Ph.D. thesis, Virginia Polytechnic Institute and State University, 2008.

${ }^{15}$ Devenport, W., Staubs, J. K., and Glegg, S. A. L., "Sound Radiation from Real Airfoils in Turbulence," Journal of Sound and Vibration, Vol. 329, 2010, pp. 3470-3483.

${ }^{16}$ Deconinck, T., Hoffer, P.-A., Hirsch, C., Meulenaere, A. D., Bonaccorsi, J.-C., and Ghorbaniasl, G., "Prediction of Nearand Far-Field Noise Generated by Contra-Rotating Open Rotors," 16th AIAA/CEAS Aeroacoustics Conference, Stockholm Sweden, No. 2010-3794, 2010.

${ }^{17}$ Hall, A. M., Atassi, O. V., and Gilson, J., "Effects of Leading-Edge Thickness on High-Speed Airfoil-Turbulence Interaction Noise," 17th AIAA/CEAS Aeroacoustics Conference, Portland, Oregon, No. 2011-2861, 2011.

${ }^{18}$ Zhang, X., Chen, X., and Nelson, P. A., "Computation of Spinning Modal Radiation from an Unflanged Duct," AIAA Journal, Vol. 42, No. 6, 2004, pp. 1795-1801.

${ }^{19} \mathrm{X}$. Wang, Hu, Z., and X. Zhang, "Aeroacoustics Effects of High-lift Wing Flat Track and Cut-out System," International Journal of Aeroacoustics, Vol. 12, No. 3, 2013, pp. 283-308.

${ }^{20}$ Liu, W., Kim, J. W., Zhang, X., Angland, D., and Caruelle, B., "Landing Gear Noise Prediction using High-Order Finite Difference Schemes," Journal of Sound and Vibration, Vol. 332, No. 14, 2013, pp. 3417-3534.

${ }^{21}$ Hainaut, T., "CAA Study of Airfoil Broadband Interaction Noise using Stochastic Turbulent Vorticity Sources," 21st AIAA/CEAS Aeroacoustics Conference, No. AIAA 2015-2222, 2015, pp. 1-18.

${ }^{22} \mathrm{Kim}$, J. W., "Optimised boundary Compact Finite Difference Schemes for Computational Aeroacoustics," Journal of Computational Physics, Vol. 225, 2007, pp. 995-1019.

${ }^{23}$ Kim, J. W., "High-Order Compact Filters with Variable Cut-off Wavenumber and Stable Boundary Treatment," Tech. rep., University of Southampton, 2010.

${ }^{24} \mathrm{Hu}, \mathrm{F}$., Hussaini, M. Y., and Manthey, J., "Low-Dissipation and Low-Dispersion Runge-Kutta Schemes for Computational Aeroacoustics," Journal of Computational Physics, Vol. 124, No. 52, 1996, pp. 177-191.

${ }^{25}$ Kawai, S., Shankar, S. K., and Lele, S. K., "Assessment of localized artificial diffusivity scheme for large-eddy simulation of compressible turbulent flows," Journal of Computational Physics, Vol. 229, No. 5, 2010, pp. 1739-1762.

16 of 17 
${ }^{26}$ Cook, A. W., "Artificial fluid properties for large-eddy simulation of compressible turbulent mixing," Physics of Fluids, Vol. 19, No. 055103, 2007.

${ }^{27}$ Kim, J. W., Lau, A. S. H., and Sandham, N. D., "CAA Boundary Conditions for Airfoil Noise Due to High-Frequency Gusts," Periodica Engineering, Vol. 6, No. 6, jan 2010, pp. 244-253.

${ }^{28}$ Kawai, S. and Lele, S. K., "Large-Eddy Simulation of Jet Mixing in Supersonic Crossflows," AIAA Journal, Vol. 48, No. 9, 2010, pp. 2063-2083.

${ }^{29}$ J. W. Kim and Lee, D. J., "Fourth Computational Aeroacoustics (CAA) Workshop on Benchmark Problems," Proceedings of Third CAA Workshop on Benchmark Problems, Vol. NACA CP-20, 2000, pp. 235-245.

${ }^{30} \mathrm{Kim}$, J. W. and Lee, D. J., "Adaptive Nonlinear Artificial Dissipation Model for Computational Aeroacoustics," AIAA Journal, Vol. 39, No. 5, 2001.

${ }^{31}$ Qian, Z. and Lee, C. H., "A class of large time step Godunov schemes for hyperbolic conservation laws and applications," Journal of Computational Physics, Vol. 230, No. 19, 2011, pp. 7418-7440.

${ }^{32}$ Atassi, H. M., Subramaniam, S., and Scott, J. R., "Acoustic radiation from lifting airfoils in compressible subsonic flow," 13th Aeroacoustics Conference, oct 1990. 